\title{
A woman with leprosy is in double jeopardy
}

\author{
AMY MORRISON \\ Medical student, Guy's St Thomas's and King's Medical School, \\ London, $U K$
}

Accepted for publication 15 March 2000

\begin{abstract}
Summary The double jeopardy associated with female leprosy patients is the central theme underpinning this essay. It constitutes a combination of biological factors unique to women and culturally defined bias, resulting in more stigmatization and isolation for women. Having examined the female immunological response and biological roles, the essay continues by focusing on the gender-culture perspective of leprosy. It draws upon an historical analysis of the experiences of Indian and African women to illustrate the ways in which gender roles impact upon health education and the utilization of health care services. Concluding comments suggest strategies that might improve female leprosy patient status, and views towards future research.
\end{abstract}

\section{Introduction}

Leprosy is one of the oldest diseases known to humankind, and for centuries people with the disease have been stigmatized. Social attitudes, both past and present, exude fear. In developed countries, leprosy is little known yet conjures up visions of deformed, fingerless beggars sitting in busy market squares or street corners in the slums of India or Africa. It is this stereotypical Western view of leprosy that emphasizes the stigma inflicted upon people with leprosy. The purpose of this essay is to examine the additional gender specific social impacts of leprosy on women. This is defined here as the 'double jeopardy'. Specifically, this definition goes further than describing leprosy stigma in general, to include both uniquely female immunological and biological involvement, and the cultural impact on the social status of women that results in the gender bias of health beliefs, attitudes and behaviours. With special reference to the link between gender and culture, the essay will analyse the emerging roles of women in Indian and African culture to provide the background for understanding the specific problems of female leprosy patients in these two broad geographical areas. In the concluding sections, a direction for health prevention and promotion that takes account of the cultural norms and boundaries of women in Africa and India will be sought. This will recognize key challenges in conducting comparative analyses that recognize

Correspondence to: A. Morrison, 47 Brixton Hill Court, Brixton Hill, Brixton, London

This article is adapted from one of the winning entries for the Lepra Essay Competition, 1999. 
the implications of eurocentric bias. The concluding analysis will, therefore, attempt to synthesize the discussion, as far as is possible, from the cultural perspectives of those studied.

\section{Leprosy and pregnancy}

Older hospital-bound data suggested that often appeared first in pregnancy, with reactivation and relapse of pre-existing disease. ${ }^{1}$ These observations do not seem to hold true now, but there are no published hard data from leprosy field programmes about pregnancy and first presentation of leprosy. Women are at particular risk of developing type 1 (reversal) reactions during the postpartum period. ${ }^{2}$ This puts women at particular risk of developing neuritis and nerve damage.

A major concern for women is the effect that leprosy may have on their children. Pregnancy in mothers with lepromatous leprosy is no more affected by the major complications of pregnancy than other forms. ${ }^{3}$ However, neonates appear to weigh less than average, and have a higher incidence of fetal distress and respiratory problems. ${ }^{3}$ This has been associated with impaired placental function, detected clinically as intrauterine growth retardation. ${ }^{4}$ These babies have a higher incidence of neonatal and infant morbidity due to an increased susceptibility to common childhood infections, and a higher incidence of neonatal death due to respiratory problems. However, these studies relate to mothers treated in the pre-MDT era and data relating to the subcore of pregnancy after MDT treatment are sadly lacking.

The possibility that leprosy is transmitted from mothers to babies in utero has been investigated; again, most of the data predate effective combination antibiotic therapy. It was discovered that cord blood IgA was significantly increased in babies of mothers with leprosy, and IgA anti-M. leprae antibodies were found in tie cord sera of $30 \%$ of these babies. ${ }^{5}$ Furthermore, evidence of active production of specific IgA and IgM anti-M. leprae antibody was found during the first 6 months of life. ${ }^{6}$ This evidence suggests that $M$. leprae crosses the placenta to set up an immune response in the child. However, the incidence of leprosy in children under 2 years of age, born to mothers with active lepromatous leprosy, was only $5 \%{ }^{7}$ These babies are at particularly high risk of infection by droplet spread because they are in close contact with mothers who have leprosy, and often share the same under-privileged social circumstances, for example, poor housing, lack of ventilation, and overcrowding. In summary, women are not only disadvantaged by the detrimental effect of pregnancy on leprosy, but also the marked disadvantages that leprosy inflicts on pregnancy and child health. The cultural impact on these biological mechanisms is discussed later.

\section{Cultural and social factors}

The double jeopardy of leprosy constitutes not only the integration of the disease with uniquely female immunological and biological influences, but also, and perhaps more importantly, the cultural impact on the social status of women that results in the gender bias of health beliefs, attitudes and behaviours. Together, these create and maintain the additional social stigma of leprosy for women. Furthermore, the biological disadvantages that cause the negative evolution of the disease are made significantly worse by the added stigma created by gender and cultural bias against women (Figure 1). In this section, the general effects of gender and culture on leprosy for women will be discussed. However, in order to understand the social effect of leprosy for women, clear knowledge of their culture must be sought. An attempt to address the 


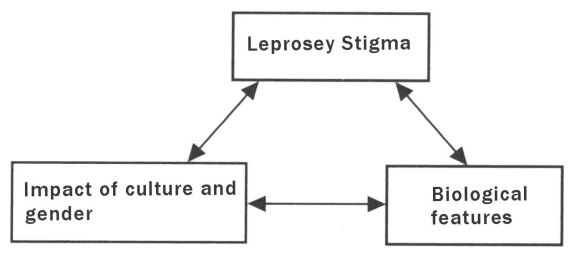

Figure 1. Components of the double jeopardy.

problem in such ways will minimize the tendency towards a eurocentric bias in ideologies, and preventative and remedial health programmes. Here, the emergence of gender bias within Indian and African cultures will be discussed to provide a backdrop for understanding the specific problems faced by female leprosy patients in these two geographical areas.

\section{Women in India}

In pre-colonial India, each religious community governed itself according to a body of local customs. The brahmins, India's highest caste, exercised the most control over women. Women were kept secluded and viewed as subordinate. Unable to marry out of caste, women served to maintain property within the caste and secure male positions in the social hierarchy. Primarily sexual control and the sexual purity of women governed the social respectability of men. The result, therefore, was a system whereby men, their family, and caste, gained social honour from women, thus reinforcing the need for women to be secluded and protected.

Indian family life today is as much governed by caste, class and gender as in the precolonial days. The political changes affecting Indian women have undoubtedly led to the formation of a modified family structure and support networks for women of higher caste and class. For those living in poverty and deprivation, namely lower castes and classes, fewer changes are to be found. Two types of family structure predominate, the nuclear family and the joint family. In the traditional joint family the new wife's primary relationship is with her mother-in-law. As a wife, a woman has obligations to her husband, her children and her mother-in-law. She is responsible, under the supervision of her mother-in-law, for domestic duties, caring for children and elderly family members, organizing rituals and festivals, and fulfilling social obligations towards friends and relatives. It is these duties that maintain the seclusion of women. From childhood, women have a socio-psychological disposition to believe that their role is within the home. In the lower social classes this structure remains. In the higher classes, where women may choose to, or be obliged to work, all these duties remain. In some families, structures may have been adapted to help the working wife and mother. Nuclear families became more common as young people moved to work in cities. Here, women have more power, but their duties remain the same, and they do not have the extended family to help them.

In brief, the preservation of male dominance in India has allowed only the minority of high social class women to strive for success. For the remainder, namely women of low caste and class, the male dominated world has been a constant for many hundreds of years. It is these women, who are uneducated, isolated, and living in poor conditions that are at higher risk of leprosy and its surrounding stigmata. 


\section{Women in Africa}

For centuries, African society has centred on consanguineal families. Here, the notion of family is based on the enduring, separate kinship ties of men and women. Loyalty to kin outweighs the bond of marriage. Consanguinity was in the form of either matrilineal or patrilineal descent, where children of the marital union belonged to the female or male line, respectively. In matrilineal descent, for example the Swahili of Tanzania, male partners would live with females' families and in patrilineal descent, for example, the Tombo village people of Sierra Leone, females would move to the family compounds of the males. In addition to consanguinity, polygamy was also part of the culture. In many areas, men could take more than one wife and often lived in their own residence separate from their wives. They took no economic responsibility for supporting their wives. This led women to subsistence farming to support themselves and their children. Perhaps this has led to the western image of black African women as strong and hardworking and provides typical images of African women working the land with babies tied to their backs. Husbands, therefore, did not guarantee security as in the Indian culture. Instead, in African society, kin could always be called upon for economic support and, in addition, it was kin who inherited land not spouses.

In the above example, women lived side-by-side as co-wives. Often the first wife was the dominant female, who looked upon new wives favourably because they added to the workforce. However, women were not always given their hierarchical level automatically, and they had the added pressure of competition over attractiveness, domestic and economic activities, as well as the need to please their mother-in-law. Together, husbands and motherin-laws exerted control over women who had to gain permission for everyday activities. Unfair treatment of women was combated by women's kin groups who had the power to pressurize men to modify their behaviours.

Women's relative power and independence in Africa began to slip away with the monetarization of the economy, urbanization, and colonial and post independence development policies. ${ }^{8}$ All led to the involvement of men in a cash economy. Women and children had less cash earning potential due to their continued subsistence needs. Women's work lost prestige and became devalued, leaving them vulnerable. In the cash earning economy rural women could no longer barter for goods, and their workload increased to earn money. Husbands continued to expect women to look after their own needs in the same way as in the pre-cash economy. Gender inequality grew as men continued to earn cash, and being unable to support themselves, women became increasingly more dependent on men. Women no longer had their own rights within marriage because they and their kin were dependent on the same men for money.

The effect of urbanization was also to fragment descent groups and the kinship ties of older neighbourhoods. Kin groups could no longer function on a daily basis. Women began to find friendships and support out of their kin groups and sometimes across ethnic divides. In many ways, women could adapt to these changes, but there were new boundaries that took away much of their independence within the urban setting. They had no right to housing, and were therefore forced to live with their husbands. This removed privacy and created new strains on the marriage. Many women insisted on civil marriage ceremonies to prevent polygamy and shared income, but men continued to take unofficial wives. In East Africa, many men feared female independence and to a large extent controlled female income. The cash earning potential of girls and women in the urban economy was, therefore, still limited. 
Female poverty, both rural and urban, created by monetarization, has left much of the female population of Africa in fear of destitution. This has led to high fertility levels to produce offspring that will provide social security by contributing to labour intensive activities. This has resulted in low levels of education, few skills, and low income, which in turn maintain high fertility rates. This vicious circle traps women in poverty and a life of male dominance.

To summarize, African and Indian women have both been moulded by their traditional nurturing roles, by colonial rule, and by western influences on their respective economies. Both groups of women are largely dependent on men and persistently bound to domestic activities and child rearing. In both continents, it is the women of the lowest social classes who have experienced the most restrictions on mobility, education, and personal finances. A critical outcome has been to engulf their lives in forms of poverty, which place them at particular risk from chronic diseases, like leprosy. Leprosy and its attendant risks, therefore, can only be studied and countered by assessing the problems and needs of these women within their own cultural backgrounds. The next section aims to address the culture and gender induced problems for women with leprosy in African and Indian society.

\section{Social and cultural dimensions of leprosy for women}

Global registered prevalence of leprosy in 1996 was 0.17 per 1000 population. ${ }^{9}$ This figure was probably an underestimate because of wide differences between registered cases and actual numbers. World wide, the number of registered patients has reduced over the last 10 years, ${ }^{10}$ but in India and Africa leprosy remained endemic. Prevalence rates in India and Africa were significantly higher than world-wide rates, at 5.9 per 10,000 population in India and from 7.79 (Central African Republic) to 0.09 (South Africa) per 10,000 in Africa. ${ }^{11}$ Case detection rates vary from country to country, but in India and most of the rest of the world, rates were higher in males compared to females, producing a ratio of 2:1. In Africa the gender ratios reflected higher base detection rates for women than men. In Kenya, the gender ratio was $1: 1,{ }^{10}$ with even higher rates for females in Uganda ${ }^{12}$ and Malawi. ${ }^{13}$ Why is there a difference in gender ratios between India and Africa? Is it that women in India are less exposed to leprosy in comparison to men than women in Africa? Do African women have less fear of stigmatization compared to women in India, leading them to seek medical care more frequently? Are medical services in Africa more accessible to women compared to India? These questions are addressed below.

\section{Exposure to leprosy}

Cultural diversity has resulted in different levels of female exposure to leprosy. In Africa, women's roles take them away from their family home. They often have sole responsibility for themselves and their children, which has led them towards subsistence farming and cash earning work in the community. In addition, African women are in contact with relatives outside their immediate family due to kinship ties. This takes them beyond their own communities to different settlements. It is this community contact that may result in more frequent exposure to leprosy.

In contrast, the role of Indian women has been within the domestic environment for many 
hundreds of years. This seclusion limits their community contacts with leprosy. However, seclusion brings its own problems in leaving leprosy cases undetected for longer. ${ }^{10}$ In countries like India, Ulrich et al. ${ }^{14}$ have attributed the decreasing incidence of leprosy and the lower case detection rates amongst women not only to seclusion alone, but also to the decreased marriage opportunities for women with leprosy This limits reproductive activity, and consequently fewer children are born into high-risk leprous environments. Where women do marry, numbers of pregnancies tend to be limited to prevent the subsequent deterioration to women's health.

Across both continents there are common exposure problems for all women in the lower socio-economic groups. Specifically, lower socio-economic groups tend to have poor housing, inadequate ventilation, low nutritional status, and higher risk of leprosy contacts. Poor nutrition, in particular, can compromise immunological status. ${ }^{14}$ Religious and culturally determined food habits can lead to specific deficiencies. In general, females have a high risk of iron deficiency anaemia, which causes increased susceptibility to disease. Intercurrent infection, more common in deprived groups, also leads to lowered immunity and a more serious risk of contracting leprosy. Female exposure to leprosy cannot, by itself, offer a full explanation of the incidence and prevalence of leprosy among women. For a fuller explanation, we need next to explore women's use of health services.

\section{Utilization of health services}

Access to and use of health services by women can be influenced in a number of ways: the availability of health care; literacy levels; awareness of the disease, and of available health care; family position and decision-making power; geographical and family mobility issues; the quality of health care provided; and the stigma associated with the disease.

The availability of health care is a critical aspect of early detection and treatment of leprosy. In 1988, the World Health Organisation Expert Committee on Leprosy recommended that leprosy control should be integrated into primary health care services. In making this recommendation, it was considered that health services would reach a greater proportion of the population. However, research from India suggests that detection rates in rural areas are much lower for women than men. In urban areas, detection rates are not significantly different between the sexes. These differences are related to lower health care coverage in rural areas. ${ }^{15}$

In combination, literacy and awareness levels have a key impact upon the utilization of health services. Specifically, if women have a poor understanding of causation, symptoms and available health care, then services, however efficient, will not reach the target population. In India, leprosy was traditionally thought to result from the 'wrath of god' ${ }^{16}$ This resulted in women delaying treatment until measures such as fasting and offerings had been made. This misconception has become less frequent, but new levels of awareness have not prevented delays in women seeking treatment. ${ }^{17}$ In general, women in India have poor knowledge of the presenting symptoms of leprosy, and are less aware of the health services available to them than men. ${ }^{18}$ It is likely that Indian women's poor knowledge of leprosy stems from a lack of education and the seclusion that is typical of Indian culture.

Similarly, African women often have a very traditional understanding of the underlying cause of leprosy. For example, it was thought that when people died, they became spirits. The spirits or 'muzimu' had to be appeased and made comfortable continuously because they became easily upset. If ignored or neglected, they could inflict leprosy on anyone, not necessarily the neglector. This belief led many African people to wear charms to ward off 
the spirits. ${ }^{19}$ 'Bad blood' reflects a modern understanding of leprosy, but is also felt to be the causative factor in sexually transmitted diseases and psychiatric illnesses. ${ }^{19}$ However, specific knowledge of causation appears to be lacking in much of Africa, including northwestern Botswana, ${ }^{20}$ Ethiopia, ${ }^{21}$ Congo and Tanzania. ${ }^{22}$

In Botswana, the clinical signs of leprosy were linked appropriately to skin lesions, ${ }^{20}$ a contributory factor to early detection and health seeking behaviour. In contrast, awareness of symptoms and availability of services is poor among Nigerian females. This has been attributed to Nigerian culture, where spouses are the most important sources of information for women about leprosy. ${ }^{23}$ However, it appears that among many African women, there is knowledge about modern healthcare availability, and there is a more universal belief that leprosy is curable. Moreover, traditional lifestyle and beliefs in African medicine have meant that modern healthcare ideas have been integrated into pre-existing health beliefs. This is particularly so for women, who are not educated to understand leprosy in a more modern way. African women are more likely to try over-the-counter remedies first, followed by traditional healers, and then health clinics, leading to delays in treatment. In brief, evidence suggests that both Indian and African women are less aware of causation, symptoms, and the availability of health care than men in each respective geographical area. This can be attributed to illiteracy and to socioeconomic deprivation in both countries, and the continued seclusion of women in India.

The decision-making powers of women are determined by their status in the family structure. Indian women, particularly of lower caste and class, tend to have few decisionmaking powers in the home, and experience low social status in the family. They are controlled by their spouses in both joint and nuclear families, and frequently in the joint family structure by their mother-in-laws. Thus, health care decisions for women are often made by husbands or mother-in-laws. Research suggests that Indian women exhibiting early signs of leprosy often delay seeking medical attention because of their partners' apathy or their mother-in-laws' jealousy. ${ }^{15}$ Delayed medical treatment can result in deterioration of the disease and a higher risk of deformity. In addition, while the disease remains active, other members of the family are at greater risk of infection.

In rural Africa, where women often have responsibility for the home, work and the upbringing of children, decision-making powers still appear to remain with men. Senior males make the majority of key decisions, including those about female healthcare. ${ }^{20}$ In contrast to the male apathy of India, beliefs that leprosy is curable have resulted in community attitudes in Africa. Such attitudes encourage women to seek early treatment. ${ }^{20}$ In brief, in much of India and Africa, the authority to make decisions regarding health care lies with men. In India, this culturally determined social framework has often hindered female leprosy patients. In contrast, evidence suggests that the attitudes of African men allow women to seek medical care more promptly.

The geographical mobility of women is limited by lack of decision-making power, time, money, an unwillingness to disrupt household duties, and the inability to find other caretakers for dependent elderly relatives and children. ${ }^{10}$ Indian women in joint families have a potential advantage here, in that they may have relatives available to care for children and other dependants. However, this advantage is undermined by their lack of decision-making power, a lack of money, and an unwillingness to disrupt household duties. Traditionally Indian women have been moulded to believe that their role is within the family home caring for others. To spend time and money seeking personal health care may involve confrontations with husbands or mother-in-laws, and guilt from leaving family uncared for. The nuclear family structure may provide less flexibility for women because they do not have additional carers in the home. In 
the higher social classes, however, women from nuclear families are more empowered, have more money available to them, and have servants to share household duties.

In Africa, the mobility of women is still restricted, but due to the cultural differences between India and Africa, the nature of the restrictions is different. African women have limited decision making powers, as outlined above. The positive attitude of their community towards seeking medical care and the practice of making alternative carers available, linked to other wives or kinship ties, offsets this. However, in terms of time and money, women have little of either. This is reinforced by their involvement in subsistence farming rather than the cash earning employment activities of men. It appears that the mobility of African and Indian women is limited, but that the limits are not the same for each group. This reflects their roles in two very different cultures.

The quality of health services available can provide major barriers for leprosy patients seeking health care. A problematic issue for females in general is the fear and embarrassment of having to see a male doctor. In western society women can choose to see female doctors if they wish, but in developing countries this service is not always available. By not catering for both sexes, health care services reflect a poor understanding of the needs of female patients. In India and parts of Africa, this lack of gender awareness is particularly problematic. The reason for this is two-fold. Firstly, a high proportion of health clinics are male dominated and secondly, women of certain religions are forbidden to see male doctors. After puberty, Islamic women are not allowed to show their bodies to any other man apart from their husband. In India, this problem may be reflected in case detection rates of males and females aged 11-17 years, where the female detection rate is half that of males. ${ }^{10}$

The stigma associated with leprosy acts as a barrier to seeking medical care. Women's fears centre on damaging their marriage prospects, losing their husband, children and homes, and inflicting the stigma associated with the disease onto their children. For men, the disease does not lead to such severe consequences. In India, women hide their symptoms for longer than men do for fear of stigmatization. ${ }^{17}$ The outcome of leprosy diagnosis and related stigma is discussed below.

To summarize, relative to men, women in India and Africa are disadvantaged in their utilization of health care services. The culturally determined roles of women lead to lower levels of awareness of the symptoms of leprosy and available medical facilities. This is further aggravated by their inferior decision making power, poor mobility and a greater fear of associated stigma. Furthermore, health care services appear to reflect this gender bias, both in availability and specific services for women. This gender discrimination in society and health care services is reflected in a survey by Rao et al., ${ }^{17}$ where $85 \%$ of female leprosy patients felt that gender bias was definitely responsible for the delay in the detection of their disease. Interestingly, $67 \%$ of men agreed with this opinion. Below, the gender inequality is discussed in relation to case detection methods.

\section{Case detection}

Case detection is the organized and systematic search for patients in the community. Its intensification can only be justified if there is adequate facilities for treating a larger group of patients. ${ }^{24}$ Case finding methods are broadly split into two types: active and passive. Passive case finding includes voluntary reporting, and referral and notification. Voluntary reporting requires community awareness of the signs and symptoms of leprosy, and an efficient and 
reliable diagnostic and treatment service. However, its efficiency as a case detection method is impaired by the intense social stigma related to leprosy. For women in particular, voluntary reporting is limited further by lack of awareness and poor mobility

Active case finding methods aim to screen populations to find unreported cases of disease. There are two major types: general surveys and contact surveys. General surveys are useful in areas of high endemnicity, but are both expensive and time consuming. They involve either systematic house-to-house enquiry, or the gathering of populations to central points in villages. The first method ensures all women are seen; the second is open to the risk of female non-attendance. Contact surveys involve the surveillance of all contacts of high risk leprosy patients. This method has been found to be successful in areas of low and moderate endemnicity, where there is greater clustering of leprosy. ${ }^{25}$ However, it only identifies a small proportion of new cases arising specific area.

Case detection methods show gender insensitivity. ${ }^{10}$ Rao et al. ${ }^{26}$ report poor detection of female cases through passive methods, and more effective detection through active case finding. This would reflect the low level of leprosy awareness amongst women, lack of mobility, and fear of stigmatization. Active case finding cuts across gender bias, ensuring female detection. There are specific case finding problems in India among females aged 11-17 years where examination by male medical staff is problematic.

Contrary to the evidence of Rao et $a .^{26}$, recommendations of the WHO Expert Committee on Leprosy ${ }^{25}$ favoured self-detection through health education. This runs counter to the above evidence, and fails to take account of gender sensitivity in case finding. In addition to detection and diagnosis, gender differences in treatment compliance, as discussed below, are important in terms of health education programmes.

\section{Treatment and compliance}

The treatment of leprosy involves administration of antibacterial drugs (rifampin, clofazimine and dapsone; MDT). The aim is to kill $M$. leprae in the shortest possible period and prevent emergence of resistant strains of $M$. leprae. ${ }^{17}$ Additional management of eye, nerve, hand and foot lesions involves physiotherapy, occupational therapy, and the ability to recognize serious manifestations of the disease for referral.

Evidence indicates that women are more compliant with drug treatment than men. ${ }^{26}$ In India, female compliance may be a result of women having been socialized to conform to prescribed behaviours. However, the benefit of female compliance is perhaps outweighed by delay in seeking medical care. ${ }^{17}$ It is interesting that Indian women often rely on traditional and spiritual healing as well as drug therapy. This may be a result of the poor understanding of treatment by women compared to men. ${ }^{27}$ African women also combine modern treatments with traditional medicine. This is satisfactory because most traditional healers do not believe that modern and traditional African medicine should be mutually exclusive. ${ }^{21}$

Although female compliance is better than male complianceto drug therapy, it is Important to note that females are not $100 \%$ compliant. Indeed. Vlassof (in Le Grand ${ }^{10}$ ) found that 1000 of female leprosy patients in a leprosy colony in India were non-compliant, despite awareness of the need for treatment. A well documented problem with female compliance in India is the similarity of monthly packets of dapsone pills to oral contraceptive pill packaging, leading to conflict with mothers-in-law who want their sons to have children. Furthermore, compliance is hindered by difficulty in attending treatment clinics due to limited time, money and transport. 


\section{Outcome of diagnosis}

Diagnosis of leprosy undoubtedly results in shock for individuals and their families. Men diagnosed with leprosy in India suffer fewer negative reactions, such as shame, fear, or blame from spouses. Women suffer from more negative reactions. Positive reactions, for example, sympathy and support, are rare for both sexes, but particularly so for women. ${ }^{10}$ The power and influence of men within the family structure enables them to maintain their position in the household more frequently than women. ${ }^{10}$ Women report being pressurized to leave the family home, but certain factors influence them to remain: strong marriages; husbands that are supportive in finding early treatment; prompt detection of the disease; seeking early treatment; and motherhood, which elevates women's status within the family. ${ }^{27}$ However, under present law, it is easy for spouses to insist on separation (Muslim Marriage Act, 1939; Special Marriage Act, 1954; and the Hindu Marriage Act, 1955). ${ }^{28}$

Where women remain with their families, they are often banned from sleeping in the same room as their spouse and family. There is a particularly high discriminatory attitude towards women in joint families. This results in a move from joint to nuclear families after detection. This is particularly common in South India. ${ }^{29}$

Family reaction to leprosy diagnosis is not as well documented in Africa. It appears that, in the early stages, individuals remain well integrated in their families. ${ }^{20}$ Later, as deformities develop, sexual relations are prohibited and divorce is easily secured. ${ }^{19}$ The literature does not specify any gender bias, but it is interesting to find that in a survey of a Zambian leprosy colony, more males than females reported that their spouses left them after diagnosis. ${ }^{19}$ This may be a result of women being less economically reliant on men in rural society than, for example, in India.

Beyond the marriage bond, the effects of leprosy diagnosis on the domestic environment of women are far reaching. Women in India often become isolated from domestic duties, especially cooking and caring for children. This has a devastating effect, since it is these roles that have come to shape women's self-identity. The freedom to touch and be touched, which acts as reinforcement for female caring roles, is also limited. This is enforced by spouses, or by women who voluntarily withdraw for fear of contaminating the rest of their family. $^{27}$

African women appear to continue day-to-day domestic and farming duties. However, when deformities appear, both roles become very difficult and families may suffer as a consequence. In Ethiopia, an unwillingness to employ or work alongside leprosy patients has been documented. ${ }^{21}$

In India, community reactions to leprosy appear to discriminate between men and women. More women than men have constraints on social activities, travelling, and attendance at religious festival celebrations. ${ }^{17}$ However, this isolation is often reinforced by women who remain aloof within the community to prevent passing the disease on to others. ${ }^{28}$ In Africa there is little evidence to suggest a gender difference in community reaction to leprosy. Moreover, patients appear to be well integrated and accepted within the social structure of the community. ${ }^{20}$ In contrast, a study in Ethiopia revealed an unwillingness to provide homes for or shake hands with people with leprosy. ${ }^{21}$ This may reflect cultural differences between African communities.

Visible deformity often leads to a much more severe level of isolation for leprosy patients. In Africa, this isolation cuts across marriage bonds and kinship ties, and women are commonly expected to dissociate themselves completely from family. ${ }^{19}$ In India, the fear 
of deformity often results in women leaving their homes and family voluntarily, because without the use of their hands to perform domestic duties, they feel they are useless and a burden. In India, deformity primarily effects the hands of women and the feet of men, reflecting their respective roles indoors and outdoors. ${ }^{10}$ In Africa, it may be that both the hands and feet of women become deformed as a result of their multiple roles.

In brief, the lower social status of females in India is causing women greater suffering as a result of leprosy, in terms of marriage, family, culturally defined roles, and isolation. For African women, gender bias in the outcome of leprosy is less well documented, but the evidence presented in this essay suggests that it exists and has detrimental effects. Below, the devastating effects of leprosy on marriage prospects are discussed, followed by the potentiating effect on leprosy of the cultural demands to produce children.

\section{Marriage and children}

Women with leprosy are less likely than men to have marriage opportunities. ${ }^{30}$ In India, women with leprosy have had to make more compromises than men when choosing marriage partners. Women often marry men who are at least as deformed as themselves; in contrast, men occasionally marry healthy females. ${ }^{27}$

The gender bias and stigma associated with leprosy goes further than affecting women themselves to involve their children. Commonly, daughters have difficulty finding marriage partners. Up to $85 \%$ of women studied ${ }^{27}$ left home to prevent their daughters from facing this discrimination.

\section{Pregnancy}

In India, it is women's duty to provide children for their husbands. Failure to do so may result in confrontations with husbands and mothers-in-law, and in some cases, separation and divorce. In Africa, children provide social security for women and higher status than other childless wives in polygamous marriages. This cultural pressure to bear children leads to deterioration in disease status and consequently more isolation from family and community. Women are therefore caught in a vicious circle: they experience isolation if they do have children and isolation if they do not.

\section{Summary}

There are cultural differences between the status of women in India and Africa. These result in differential levels of exposure to leprosy, differences in understanding about causation, and a range of male attitudes towards female uptake of health care services. However, the low socioeconomic status and gender bias towards female leprosy patients also means that there are important similarities in both cultures. Specifically, women have a poorer understanding of disease causation, knowledge about symptoms, and information available on health care than men. They are reliant on men for permission to seek medical care, and have limited mobility. They suffer more discrimination from spouses, family, work and the community. In addition, women the added biological and cultural disadvantages placed on disease status by 


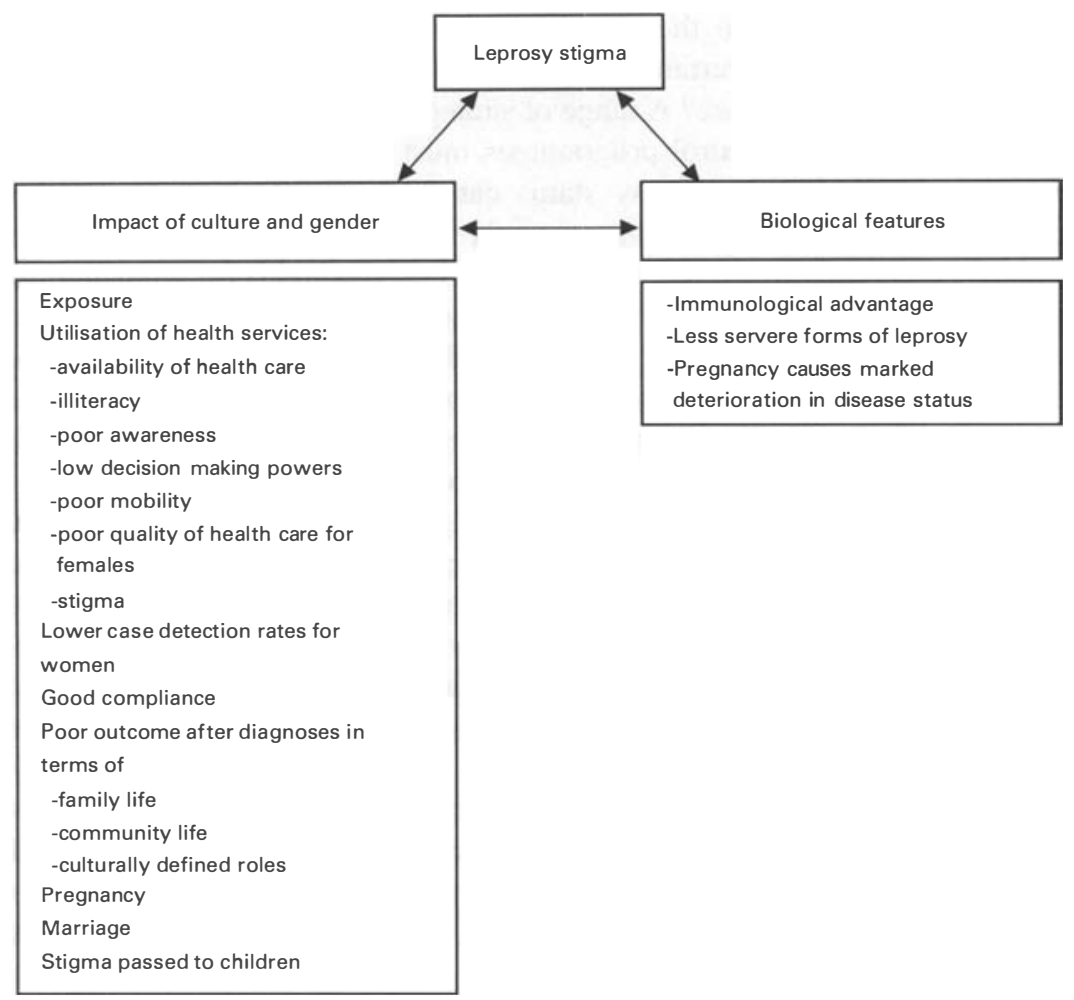

Figure 2. Components of the double jeopardy, expanded in light of discussion.

pregnancy. In brief, gender bias in India and Africa results in greater suffering for women with leprosy. This is reflected in the female under-utilization of health services, and the stigma and isolation specific to women that result from leprosy diagnosis. Furthermore, health services appear to reflect the gender bias in both the quality of services available and in case detection methods.

For Indian and African women with leprosy, the double jeopardy, therefore, is a combination of biological factors unique to women with a culturally defined gender bias, that results in more stigmatization and isolation for women (Figure 2). The culture and gender perspective therefore has very important implications for health prevention and promotion programmes.

\section{Implications for health care}

The evidence presented in this essay highlights the need to incorporate culture and gender perspectives into the health education and management programs for leprosy. Primarily, an approach based on cultural dynamics will promote better acceptance and an improved uptake of services. Moreover, a programme that incorporates gender specific issues for females will, theoretically, lead to improved detection rates and earlier treatment for women. This could be addressed at a number of levels: at the level of the individual - in terms of health beliefs; at the level of the family - linked to attitudes; and at the level of the community - in terms of local 
beliefs. Improved strategies could then be targeted at health care availability; the gender insensitivity in health services; government policies; and health budget allocations. What would such a vision of health care look like? A range of strategies is needed to support such a vision.

First, the aims of leprosy control programmes must be defined before any recommendations about improving female leprosy status can be made. Leprosy prevention is the ultimate goal of control programmes, but is presently unobtainable due to the lack of effective immunization and continued world poverty. The next best method is for women to recognize their symptoms earlier, present at health clinics sooner, comply with treatment and prevent the emergence of disabilities. The evidence presented in this essay suggests that women are more disadvantaged than men in all these activities, except for compliance. Implicated in the culturally defined inferior status of women are higher rates of illiteracy, immobility, stigmatization, and a lack of gender sensitivity within health care.

Second, at the community level, the single most effective method of improving the rates of female leprosy detection and the accompanying prevention of associated social and physical disabilities is health education. Health education aims to promote a reasonable attitude towards leprosy that neither exaggerates nor minimizes the dangers of the disease. ${ }^{24}$ It involves individuals, spouses, families, communities and health care professionals. Who provides health education? The answer is that all health care workers should be involved in health education, and themselves need to be given a wider understanding of leprosy and, in particular, the specific problems faced by female leprosy patients. Indeed, gender sensitivity in dealing with leprosy patients must be encouraged amongst health professionals at all levels. This includes physicians, social workers, paramedics, general medical doctors, community nurses, midwives and personnel dealing specifically with leprosy, for example, specialist doctors and leprosy nurses.

Third, health education should focus on educating women about leprosy in a culturally sensitive way, with specific objectives that will enable them to seek medical help, and reduce social stigmatization. Women need to be taught a number of fundamental facts about leprosy in order to undermine any preconceived ideas that could hamper its detection or women's attendance at health clinics. First and foremost, women must be able to recognize the presenting symptoms of leprosy, know that it can be cured with early treatment, and know where to go to get the treatment they require. Additionally, information about the causation of leprosy will provide a better understanding of the disease. After diagnosis, health education should centre on issues of compliance, deformity prevention and pregnancy. The biggest problem in educating women appears to be accessing them. Many methods have been suggested, for example community lectures by health workers, informative newspaper articles, talks on the radio, posters, pamphlets, booklets and film strips. All of these methods, however, require either literacy or mobility Other methods that may be more accessible to women include the formation of women's groups within villages with female teachers or female members of local government as educators. ${ }^{17}$ It may also be possible to build upon female reliance on traditional medicine and religion by educating local healers and religious leaders to detect and refer leprosy cases: and to educate women about leprosy. The paramount issues, therefore, in health education for women are to make it accessible, understandable, and non-threatening. Once leprosy is diagnosed, health workers can continue to educate women about leprosy during their attendance at clinics for treatment, family visits and community talks.

Fourth, the health-education of women alone may not be enough to overcome the mobility problems or stigmatization specific to women. Specifically, lack of mobility and 
poor decision-making powers can inhibit women from seeking care. Critically, it is not only women who require this type of education, but also their spouses and families. For example, if spouses have an understanding of the early symptoms of leprosy and the need for prompt treatment to promote cure, then they are more likely to allow women to seek health care. Family understanding could also help women to follow complicated drug regimens, and could encourage patients towards treatment compliance. Indeed, the real importance of family empathy may be in discouraging women from hiding their disease for fear of isolation and stigmatization.

Fifth, whole communities must be targeted with regard to health education if the stigmatization associated with female leprosy is to be reduced. It is the adverse reactions of communities to female leprosy patients that leads to the devalued status and social rejection of women. If local and national communities were to understand the cause, ease of treatment, and the consequences of a lack of community empathy, then perhaps women would feel less afraid of approaching leprosy health care services. It has been suggested ${ }^{1}$ that the most effective method of reaching communities would be to educate all school age children. From this perspective, education is the key to developing societies which may, in the future, be less stigmatizing and more accepting. In addition to increasing community awareness of leprosy, opportunities to mobilize community resources and strengthen community co-operation are more likely to facilitate early recognition and to promote selfreporting by women. Where self reporting is ineffective, community willingness to carry out general or case contact surveys might be sought.

Health education is one of the ways of reducing gender bias associated with leprosy, and in turn, improving female detection rates. However, other strategies may need to be considered. Adapting existing health care services in order to achieve better levels of care for women with leprosy may also be important. Specifically, the education, clinical training, and employment of more female health care professionals would dilute the gender insensitivity of health care services. Women would feel more able to consult with, and be examined by other women. Indeed, the provision of female doctors may improve the poor detection rates of pubertal girls in India and parts of Africa. Once diagnosed, patients need to continue attending clinics for treatment. This causes particular problems for women due to poor mobility and little money. To respond, clinics must become more flexible to female needs, and provide treatment in places and at times convenient to women. If they fail to do so, compliance will not be maintained.

The stigmatization inherent in seeing doctors who care for leprosy patients can be dealt with at the community level with health education, but a shorter term and more immediate solution to this problem has been suggested. ${ }^{27}$ It is proposed that doctors within primary health care systems should inquire about the condition of women's skin when trying to detect leprosy. It is suggested that this approach would be more acceptable to women, the hope being that the diagnosis and treatment of' skin disease would incur less rejection and isolation. This method may help women avoid the stigma associated with leprosy, but due care would need to be taken to continue to reduce leprosy discrimination within the community through the health education strategies discussed previously.

Finally, attention needs to be given to health education and management of female leprosy patients, specifically with regards to pregnancy. As discussed earlier, pregnancy is a very vulnerable time for women with leprosy, and there are risks of permanent nerve damage. Women need to be encouraged in control of their leprosy with MDT before considering having children. This may require the adoption of locally acceptable forms of contraception. 
MDT should be continued throughout pregnancy, and women observed during pregnancy and lactation. In addition, health education is required to inform women of the signs and symptoms related to the reactions that can occur during these times. Help could also be provided to improve the survival chances of babies, thus enabling women to achieve their desired family size with fewer pregnancies. ${ }^{5}$ In brief, women with leprosy require special attention before, during, and after pregnancy to prevent the lowering of disease status. Duncan ${ }^{4}$ concludes that physicians dealing with such women should become more leprocentric, and less obstetrically minded.

Despite the limitations to change that are perpetuated by the existing economies of the developing countries where leprosy is endemic, employing health education and improved management plans can help to reduce the culturally defined, gender-specific disadvantages for female leprosy patients.

\section{Conclusion}

The culturally defined lower status of women in India and Africa has led to lower general awareness of the disease amongst women, poor utilization of health services, gender insensitivity in health care provision, and greater suffering for women with leprosy as a result of social stigmatization. The double jeopardy, therefore, has its roots in the culturally defined status and biological roles of women. In view of this, national leprosy control programs must continue to be very sensitive to the cultural backdrop of women, whilst at the same time encouraging women to attend health clinics by promoting health education and gender sensitivity with in health care. Indeed, one of the limitations of writing an essay that compares two continents is that it is very easy to generalize about culture across large geographical areas. It is important to note that there are marked intra-continental, as well as inter-continental differences between the experiences of women in India and Africa. This essay cannot hope to be precise enough to incorporate these differences, but aims to emphasize the general importance of the culture-gender perspective within leprosy research and control programmes.

Future research must look very specifically at the leprosy status of women with regard to individual cultures. It would also be interesting to investigate whether there is a gender bias associated with attitudes of the general public towards leprosy. Research that suggests that there is a greater female empathy and understanding towards leprosy would provide further evidence towards the need for female involvement in health care provision for leprosy, thus enhancing the female contribution to the expanding and developing societies that constitute India and Africa.

\section{References}

${ }^{1}$ Duncan ME, Pearson JMH, Ridley DS et al. Pregnancy and leprosy: the consequences of alterations of cell mediated immunity during pregnancy and lactation. Ind J Lepr, 1982; 50, 425.

2 Lockwood DNJ, Sinha H. Pregnancy and leprosy: a comprehensive literature review. Int J Lepr, 1999; 67: 6-12.

3 Duncan ME. Babies of mothers with leprosy have small placentae, low birth weights and grow slowly. Br J Obstet Gynaecol, 1980; 87: 471.

4 Duncan ME. An historical and clinical review of the interaction of leprosy and pregnancy: a cycle to be broken. Soc Sci Med, 1993; 37: 457-472.

5 Melsom R, Harboe M, Duncan ME et al. IgA and 1gM antibodies against Mycobacterium leprae in cord sera and in patients with leprosy: an indicator of intrauterine infection in leprosy. Scand J Immunol, 1981; 14: 343. 
${ }^{6}$ Melsom R, Harboe M, Duncan ME. IgA, $\operatorname{lgM}$ and IgG anti-M. leprae antibodies in babies of leprosy mothers during the first two years of life. Clin Exp Immunol, 1982; 49: 532.

7 Duncan ME, Melsom R, Pearson JMH et al. A clinical and immunolgical study of four babies of mothers with lepromatous leprosy, two of whom develop leprosy in infancy. Ind J Lepr, 1983; 51: 7.

8 Potash XX. Women in the changing African family. In: Hay MJ, Stichter S (eds) African women, south of the Sahara. Longman Group, 1995.

9 World Health Organisation. Weekly Epidemiol Rec (May), 1996.

${ }^{10}$ Le Grande A. (1997) Women and leprosy: a review. Lepr Rev, 1997; 68: 203-211.

11 World Health Organisation. Action Programme for the Elimination of Leprosy, status report, updated 1997, WHO/ LEP/97.4. WHO, Geneva, 1997.

12 Fine PEM. Leprosy: the epidemiology of a slow bacterium. Epidemiol Rev, 1982; 3: 161

13 Nordeen S.K. The epidemiology of leprosy. In: Hasting RC (ed) Leprosy. Churchill Livingstone, Edinburgh, 1985.

14 Ulrich M, Zulveta AM, Caceres-Dittmar G et al. Leprosy in women: characteristics and repercussions. Soc Sci Med, 1993; 37: 445-456.

15 Mull JD, Shearwood C, Gan LP, Mull DS. (1989) Culture and compliance among, leprosy patients in Pakistan. Soc Sci Med, 1989; 29: 799.

16 Vlassoff C, Rao S, Garole V et al. The family: a neglected determinant of health in South Asia. In: Le Grande A (1997).

17 Rao S, Garble V, Walawalkar S, Khot S, Karandikar N. Gender differentials in the social impact of leprosy. Lepr Rev, 1996; 67: 190-199.

18 Crook N, Ramosubban R, Samy A, Singh B. An educational approach to leprosy control: an evaluation of knowledge, attitude and practice in 2 poor localities in Bombay, India. Lepr Rev, 1991; 62: 395-401.

19 Holmes CJ. The self image of leprosy patients: a study of a missionary hospital in Zambia. Unpublished dissertation, University of Leicester, 1980.

${ }^{20}$ Kumaresan JA, Maganu ET. Socio-cultural dimensions of leprosy in north-western Botswana. Soc Sci Med, 1994; 39: 537-541.

21 Teckle-Haimanot R, Forsgren L, Gebrre-Mariam A et al. Attitudes of rural people in central ethiopia towards leprosy and a brief comparison with observations on leprosy. Lepr Rev, 1992; 63: 157-168.

22 Van Etten GM, Anten JG. (1972) Evaluation of health education in a Tanzanian leprosy scheme. Int J Lepr, 1972; 40: $402-409$.

23 Awoteso N. Effects of socio-cultural beliefs on patients perceptions of leprosy. Trop Geogr Med, 1995; 47: 175178.

24 World Health Organisation. A guide to leprosy control, 2nd edition. Geneva, 1988.

25 World Health Organisation Expert Committee on Leprosy. Sixth report. Geneva, 1988

26 Rao S, Khot S, Walawalkar S, Garole V, Karandikar N. In: Le Grand A (1997).

27 Vlassoff C, Khot S, Rào S (1996) Double jeopardy: women and leprosy in India. World Health Statist Q, 1996; 49: $120-126$.

${ }^{28}$ Kaur H, Ramesh V. Social problems of women leprosy patients - a study conducted at two urban leprosy centres in Delhi. Lepr Rev, 1994; 65: 361-375.

29 Ramu G, Bwivedi MP, Lyer CGS. Social reaction to leprosy in a rural population in Chingleput district (Tamil Nadu). Lepr Ind, 1975; 47: 156-169.

30 Valencia LB. (1983) Socio-economic research in the Philippines with special references to leprosy. SE Asian Trop Med Publ Health, 1983; 14: 29. 